\title{
1 Osmolarity-regulated swelling initiates egg activation in Drosophila
}

2 Anna H. York-Andersen, Benjamin W. Wood, Elise L. Wilby, Alexander S. Berry and Timothy T.

3 Weil $^{\ddagger}$

4 Department of Zoology, University of Cambridge, Downing Street, Cambridge, CB2 3EJ, UK

$5 \quad \ddagger$ Author for correspondence (e-mail: tw419@cam.ac.uk)

\section{ABSTRACT}

7 Egg activation is a series of highly coordinated processes that prepare the mature oocyte for embryogenesis. Typically associated with fertilisation, egg activation results in many downstream outcomes, including the resumption of the meiotic cell cycle, translation of maternal mRNAs and cross-linking of the vitelline membrane. While some aspects of egg activation, such as initiation factors in mammals and environmental cues in sea animals, have been well-documented, the mechanics of egg activation in insects are less well understood. For many insects, egg activation can be triggered independently of fertilisation. In Drosophila melanogaster, egg activation occurs in the oviduct resulting in a single calcium wave propagating from the posterior pole of the oocyte.

Here we use physical manipulations, genetics and live imaging to demonstrate the requirement of a volume increase for calcium entry at egg activation in mature Drosophila oocytes. The addition of water, modified with sucrose to a specific osmolarity, is sufficient to trigger the calcium wave in the mature oocyte and the downstream events associated with egg activation. We show that the swelling process is regulated by the conserved osmoregulatory channels, aquaporins (AQPs) and DEGenerin/Epithelial $\mathrm{Na}^{+}$ (DEG/ENaC) channels. Furthermore, through pharmacological and genetic disruption, we reveal a concentration-dependent requirement of Trpm channels to transport calcium, most likely from the perivitelline space, across the plasma membrane into the mature oocyte.

Our data establishes osmotic pressure as the mechanism that initiates egg activation in Drosophila and is consistent with previous work from evolutionarily distant insects, including dragonflies and mosquitos, and shows remarkable similarities to the mechanism of egg activation in some plants. 


\section{INTRODUCTION}

31 Egg activation is a conserved process that prepares a mature oocyte for embryogenesis. 32 It actuates many essential cellular processes including the resumption of meiosis, 33 modification of the outer membrane, post-transcriptional regulation of maternal mRNAs and broad changes in the cytoskeletal environment [1-3]. This process requires a transient increase of intracellular calcium, often referred to as a calcium wave(s), with multiple waves observed in mammals and ascidians, compared to a single wave in Xenopus laevis, Danio rerio and Drosophila melanogaster [4-6].

Species variation is also documented in the initiation mechanism and source of calcium required for the cytoplasmic rise [1,4]. In vertebrates and some invertebrates, egg activation is dependent on fertilisation in which sperm entry introduces Phospholipase C enzymes generating a calcium efflux from the endoplasmic reticulum [2,7]. Comparatively, egg activation in other invertebrates can be independent of fertilisation and initiated by external factors [8]. For example, the ionic composition of the solution external to the oocyte is required in the starfish Asterina pectinifera, as chelation of sodium ions in seawater disrupted the resumption of meiosis $[9,10]$. While in the shrimp Siconia ingentis, egg activation requires the presence of magnesium ions in seawater [11]. Interestingly, in the stick insect Catrausius morosus, exposure of the oocyte to oxygen in the air results in the resumption of meiosis [12].

An alternative external cue of egg activation is the application of mechanical pressure on the oocyte plasma membrane exemplified by the eggs of the wasp Pimpa turionellae, which are activated when squeezed through a polythene capillary [13,14]. This physical stress is proposed to displace the maternal nucleus and result in the resumption of the cell cycle. Similarly, the eggs of Drosophila mercatorum are thought to be activated by the pressure from the genital ducts [12]. Tension in the plasma membrane can also be generated by a change in the osmolarity of the external solution (which we will refer to as 'osmotic pressure' henceforth). Prior to egg activation, the hypertonic environment in the ovaries is thought to maintain the oocytes in a meiotically-arrested state $[15,16]$. Subsequent entry of the oocyte into a hypotonic environment results in the egg activation of dragonfly, mayfly, turnip sawfly and yellow fever mosquito eggs [16-18]. For instance, upon entry into water, yellow fever mosquito oocytes undergo a visible darkening due to the increased production and cross-linking of the endochorion at egg activation $[19,20]$. 
Overall, physical pressure appears to be a conserved mechanism for initiating egg activation in many insects.

Similar to other insects, egg activation in Drosophila melanogaster is independent of fertilisation and occurs during the passage of the mature oocyte through the oviduct [21]. One model suggests that the pressure exerted by the oviduct on the oocyte upon entry initiates egg activation [8,22]. However, more recent work has shown that external pressure alone is not sufficient to trigger a calcium wave [5,23]. An alternative model proposes that osmotic pressure generated by uptake of oviduct fluid leads to the initiation of egg activation [15]. This is supported by observations that oocytes are visibly dehydrated whilst in the ovaries, but upon deposition appear turgid and hydrated [15,24]. Rehydration at egg activation can be recapitulated ex vivo through the addition of a hypotonic solution, known as Activation Buffer (AB), which when added to an isolated mature egg results in swelling and a single calcium wave $[5,6,15]$. This influx of calcium requires the Trpm mechanosensitive channel in the plasma membrane and results in the activation of Plc21C that sustains the wave $[8,25,26]$. Regulation of calcium entry was hypothesised to be related to distribution of the Trpm protein in the membrane, as calcium entry is often seen first at the poles. However, when observed using CRISPR-generated GFP-tagged Trpm, an even distribution of the protein across the plasma membrane was evident [27]. Therefore, the precise mechanisms of initiation and regulation of the calcium wave remain to be elucidated in Drosophila.

Here, we use live imaging in conjunction with novel physical manipulation, pharmacological disruption and genetics, to demonstrate the requirement of osmotically induced swelling for calcium entry and downstream events of Drosophila egg activation. We show that depletion of osmoregulatory machinery, including AQPs and DEG/ENaC channels, disrupts water homeostasis and egg activation. We provide further evidence that the movement of calcium ions into the egg is sensitive to levels of functional Trpm. Our data also argues that the external environment is not the source of calcium for the wave, but rather the ions are likely to originate from the perivitelline space. Together with other recent work in the field, our findings reveal that Drosophila egg activation has striking mechanistic similarities to other animals and even some plants. 


\section{RESULTS}

\section{Swelling is required for the initiation and propagation of the calcium wave}

The likely initiation cues for the calcium wave at Drosophila egg activation include physical pressure applied on the posterior pole by the oviduct or the uptake of the fluid by the mature oocyte from the oviduct $[8,15]$. Our previous work has shown that physical pressure applied to the posterior pole is not sufficient to initiate the calcium wave [5]. This evidence, together with the observation that the mature oocytes are dehydrated whilst in the ovary but are turgid by the time they are deposited [15], suggests that swelling might play a role in the initiation and the propagation of the calcium wave at egg activation.

In addition to the initiation of the calcium wave, ex vivo dissected mature oocytes show an increase in oocyte volume, rounding of the oocyte poles and movement of the dorsal appendages following exposure to $A B$ (Figure 1A). To test if this swelling is required for the initiation and propagation of the calcium wave, we blocked the ability of the egg to swell by placing the anterior pole in a plastic capillary with the posterior pole being exposed to oil (Figure 1B). Upon the addition of $A B$, the oil is displaced and the calcium wave initiated as normal. However, the wave did not propagate past the opening of the capillary (Figure 1B'). An uninhibited calcium wave would normally encompass the whole oocyte by 3.5 minutes [5]. However, in this case, the wave did not propagate until the egg was expelled from the capillary. When the whole oocyte was placed in the capillary, as expected, the calcium wave did not initiate upon the addition of $A B$ (data not shown). This strongly suggests that swelling is required for the initiation and propagation of the calcium wave.

Our previous work has shown that local pressure or injection of calcium gives a localised calcium increase, but not a prolonged or broad calcium increase in a form of a wave $[5,23]$. To test if a localised internal increase in volume could induce a broad calcium event, we used a microneedle to inject halocarbon oil into a mature egg chamber mounted in halocarbon oil. Initial puncturing of the egg chamber resulted in a localised increase in calcium consistent with our previous results (Figure $1 \mathrm{C}, \mathrm{t}=0^{\prime}$ ). Injection of oil into the centre of the egg chamber resulted in a broad posterior calcium increase (Figure $1 \mathrm{C}, \mathrm{t}=$ $\left.1^{\prime}, 2^{\prime}\right)$. This response is noticeably different from previous experiments where oocytes were manipulated with a microneedle or had physical pressure applied. This data suggests swelling is necessary for the calcium propagation and is sufficient for the initiation of a broad calcium increase. 


\section{Osmotic pressure initiates the calcium wave}

126 In order to further test the function of swelling, we established a classification system that

127 enabled us to categorise calcium events in the egg and quantify our data under different 128 experimental conditions. We have classified the calcium increase exhibited as four 129 distinct phenotypes: full wave, cortical increase, partial wave, or no wave. The most 130 common phenotype is the full wave that initiates from the posterior pole and propagates 131 across an entire oocyte. This is the standard event that we observe with ex vivo egg 132 activation using $\mathrm{AB}$. We do observe a small percentage of full wave phenotypes that 133 initiate from the anterior pole. Different to a full wave, an increase in calcium can occur 134 from multiple places around the cortex. This cortical increase phenotype was originally observed when egg chambers were exposed to distilled water [5]. These observations suggest that all parts of the egg have the capacity to allow calcium into the cell and that there is a regulatory mechanism to control calcium entry. In contrast, the partial wave

138 phenotype describes the calcium wave that initiates from a pole but does not propagate 139 across the entire oocyte and recovers prematurely. We do observe some egg chambers 140 attempting to initiate waves multiple times, without successful propagation of calcium. 141 Finally, the no wave phenotype describes an absence of a calcium increase anywhere in 142 the egg for the length of the experiment. We observe this in a small percentage of eggs 143 that are likely to have a major defect in development prior to dissection.

144 Our data indicates the requirement of swelling for the calcium wave to occur at egg 145 activation (Figure 1B). One way the egg could undergo swelling is by exposure to a 146 hypotonic solution, which would cause an influx of water and subsequently generate 147 osmotic pressure within the mature oocyte. To test whether or not the uptake of water 148 alone could act as an initiation cue for the calcium wave at egg activation, ex vivo egg 149 chambers were treated with a sucrose and water solution (SW) of the same solute content 150 as $A B$, measured in osmolarity (260 mOsm). The SW solution has no ions added and is 151 very different from other buffers used to activate eggs. Sucrose is highly soluble in water 152 and is neutrally charged making it suitable for varying the osmolarity of the solution. Upon 153 the addition of SW, the egg chambers exhibited a similar proportion of the calcium wave 154 phenotypes to AB (Figure 2A).

155 To further test whether the osmolarity of an external solution is important for the initiation 156 of an internal calcium increase, egg chambers were exposed to a single SW solution from 157 a range of osmolarities. The highest percentage of full calcium waves was observed at 
$158350 \mathrm{mOsm}$, with this percentage declining rapidly by $570 \mathrm{mOsm}$ (Figure 2A'). The highest 159 proportion of cortical increases was detected at 0 mOsm (Figure 2A'), consistent with 160 predictions that an excessive volume increase cannot be regulated by the egg and results 161 in an uncontrolled calcium increase. The partial and no wave phenotypes became more 162 predominant with an increase in the osmolarity. This suggests that high osmolarity 163 solutions do not increase the internal volume that is required for the egg to complete a 164 calcium event. Together, these findings suggest that a controlled amount of water 165 entering the egg is important for regulating a calcium event at egg activation.

\section{Osmotic pressure results in the resumption of the meiotic cell cycle}

167 Previous work has shown that the addition of $A B$ to mature egg chambers can initiate 168 major cellular events associated with Drosophila egg activation, including the resumption 169 of the cell cycle and $\mathrm{P}$ body dispersion $[5,28]$. In a non-activated oocyte, the meiotic spindle is parallel to the cortex and is observed near the base of the dorsal appendages 171 at the anterior pole [28-30]. Upon egg activation, the spindle undergoes a morphological 172 change within 10 minutes, marking the resumption of the cell cycle [28].

173 To address whether osmotic pressure alone results in this change, we used Jupiter174 mCherry to label the meiotic spindle and exposed these mature egg chambers to SW 175 solution (260 mOsm). Before exposure, the spindle is a narrow ellipse with dark regions 176 in the middle where the DNA resides (Figure 2B,C,D). Upon addition of $S W$ and $A B$ 177 (positive control) the spindle shows a significant increase in width $(70 \%)$, which is 178 indicative of spindle contraction at Anaphase I (Figure 2B',C',E). When treated with 179 Schneider's Drosophila Medium (negative control) the spindle did not undergo any 180 detectable morphological change (Figure 2D',E). Together, this supports the conclusion 181 that water uptake and subsequent internal pressure is sufficient to initiate the Metaphase 182 I-to-Anaphase I transition of the meiotic spindle.

183 To further verify the role of osmotic pressure we investigated the dispersion of $\mathrm{P}$ bodies, 184 an established hallmark of egg activation [5,31]. When mature egg chambers expressing 185 a conserved $\mathrm{P}$ body marker are exposed to SW (260 mOsm), we observe a normal 186 dispersion phenotype (Figure 2F-F'). Taken together, this data suggests that an increase 187 in internal volume caused by osmotic pressure triggers downstream events of egg 188 activation. 


\section{Water homeostasis is required for egg activation}

190 The increase in internal volume observed in osmolarity experiments is regulated by water

191 influx and efflux. To test if water homeostasis is required to regulate swelling in mature oocytes, we explored the role of the water-pore channels, AQPs, which are known to coordinate the movement of water molecules [32,33]. By adding copper sulfate (a broad $A Q P$ channel antagonist) into $A B$ we do not observe a calcium wave (Figure 3A).

195

There is only one AQP channel, Prip, that is known to be expressed in the Drosophila ovarian tissue (Drosophila Fly Atlas). To investigate the role of Prip at egg activation we used knock-down tools in heterozygous deficiency or mutant backgrounds since the homozygous mutant was lethal. Upon the addition of $A B$, the number of oocytes showing a calcium wave significantly decreased in egg chambers expressing various AQP deficient backgrounds (Figure 3A). We further investigated the effect of Prip disruption by observing the time series of ex vivo activated eggs and found that half of these eggs rupture and leak cytoplasm shortly after the addition of AB (Figure 3B). Interestingly, some eggs were still able to initiate and propagate a calcium wave despite rupturing. This data is strongly suggestive of a subsequent requirement for Prip in mediating water homeostasis at egg activation.

Similar effects were observed in egg chambers expressing reduced levels of rippedpocket (rpk), a member of the mechanosensitive channels family DEG/ENaC known to be involved in transducing changes in osmotic pressure [34-36]. When activated, these egg chambers show a cortical calcium increase, rupture of the plasma membrane and leaking of the cytoplasm (Figure 3C). This phenotype is similar to when eggs are exposed to low osmolarity solutions (Figure $2 \mathrm{~A}^{\prime}$ ), suggesting that rpk is required to mediate water entry. Together, this data suggests the role of AQP and DEG/ENaC channels is to coordinate optimal swelling and water homeostasis at egg activation.

\section{External calcium is not required for initiation and propagation of the calcium wave}

In many animals, external and/or internal calcium is required for the calcium rise at egg activation [4]. To investigate the source of calcium at Drosophila egg activation, ex vivo mature egg chambers were treated with $A B$ containing the calcium chelator BAPTA. These eggs exhibited typical swelling and a full calcium wave (Figure 4A), suggesting that external calcium from the surrounding solution is not required. To further validate this experiment we depleted internal calcium by pre-incubating egg chambers with 
221 membrane-permeable BAPTA-AM (with solubilising agent PF-127). The addition of AB 222 with this chelator significantly reduced the number of calcium events (Figure 4A). 223 Together, these findings point towards the source of calcium residing within the mature 224 egg chamber.

225 There are several potential internal calcium sources in the mature egg chamber, including 226 the perivitelline space surrounding the mature oocyte [22]. The perivitelline space has 227 been shown to consist of different ions, including calcium in the early Drosophila embryo 228 [37]. However, it remains technically not possible to extract this fluid from the mature egg 229 chamber due to the dehydrated morphology. One candidate, previously shown to be 230 involved in coordinating the entry of calcium from the perivitelline space into the oocyte, 231 is the mechanosensitive channel Transient Receptor Potential M (Trpm) [26].

232 To further investigate the role of Trpm, we utilised a transgenic line from the Berkeley 233 Drosophila Genome Project which is a transposon P-element insertion in the 39th splice 234 site which results in an imprecise deletion of three exons of Trpm [38,39]. Upon the 235 addition of $A B$, these mature egg chambers swelled as expected, but fail to initiate a 236 calcium increase (Figure 4B). We further tested the requirement of Trpm using a germline 237 RNAi, which in heterozygous egg chambers resulted in a significant reduction in calcium 238 waves (Figure 4B).

239 Together, this suggests that there could be a concentration-dependent response of Trpm. 240 To test this hypothesis, egg chambers wild-type for Trpm were incubated with different 241 concentrations of Carvacrol, a broad Trpm inhibitor [40]. The addition of AB with Carvacrol 242 resulted in a significant decrease of the number of eggs with a calcium wave at a range 243 of concentrations (Figure 4C). These data support the findings that Trpm is involved in 244 regulating the entry of calcium into the mature oocyte at egg activation. Since Trpm 245 channels are located in the cell membrane [26], it is likely that their role is to allow calcium 246 from the perivitelline space to enter the oocyte at activation. 


\section{DISCUSSION}

\section{Model of Drosophila egg activation}

249 In summary, our data shows that the calcium wave and characteristic downstream events 250 associated with egg activation are initiated by osmotic pressure generated by the uptake 251 of external fluid. We show that $A Q P$ and $D E G / E N a C$ channels are required for mediating water homeostasis to withstand the rise in osmotic pressure during egg activation. We present complementary evidence that the Trpm channel is required for the influx of calcium, which we show is not supplied from a source outside of the egg chamber.

Together with previous work, our data supports the following model of Drosophila egg activation (Figure 5): (1) at ovulation, the meiotically-arrested mature oocyte passes into the lateral and then common oviduct; (2) the mature oocyte then takes up fluid due to the difference in osmolarity between the oviduct fluid and the ooplasm; (3) the increase in volume results in tension at the plasma membrane and dispersion of the cortical actin; (4) decreased density of cortical actin at the poles, prior to dispersion, primes these regions for calcium entry; (5) calcium enters the egg from the perivitelline space through the mechanosensitive Trpm channels in the plasma membrane; (6) starting at the posterior pole, further increase in intracellular calcium is relayed across the oocyte by the opening of the neighbouring Trpm channels via the dispersion of the cortical actin cytoskeleton at the lateral sides, resulting in the calcium wave propagation across the oocyte. (7) The calcium wave is then followed by an F-actin wavefront, which ensures the reorganisation of the actin cytoskeleton; (8) intracellular calcium returns to basal levels, likely through channels that transport calcium back into the perivitelline space. Collectively, the single calcium wave prepares the oocyte for pronuclear fusion and embryogenesis.

271 Osmotic pressure is a common mechanism for a volume increase and a rise in 272 intracellular calcium levels, exemplified by intestinal epithelial cells, human osteoblast273 like cells, rat astrocytes and cancer cell lines [41-44]. It is hypothesised that cells sense an increase in cell volume via intracellular solute, membrane-bound and/or cytoskeletal sensors [45]. The application of osmotic pressure seems to be a conserved initiation cue for egg activation in insects. Previous work has shown that the immersion of the oviposited mature oocytes of the yellow fever mosquito into water can resume oocyte development [46]. Similarly, for oocytes of the turnip sawfly and the malaria vector mosquito, egg activation can be initiated by placing the oocytes into water $[16,17]$. 
280 Drosophila is currently the only example of an insect in which the mature oocytes have 281 been shown to exhibit an increase in intracellular calcium in response to the addition of 282 hypotonic solution [5,6]. Our results presented here show that osmotic pressure acts as 283 the initiation cue of the calcium wave at Drosophila egg activation.

\section{AQP and Rpk requirement in water homeostasis}

285

It is essential to regulate cellular volume in response to changes in osmotic pressure. This is often achieved by AQPs, a conserved channel known to control the influx and efflux of water during cellular processes, including cell migration, neuroexcitation and epithelial fluid transport [32]. In Drosophila, our findings show the AQP Prip is required to maintain an optimal volume change at egg activation. In a Prip depleted background we observed eggs initially swelling but rupturing shortly after. This phenotype suggests that Prip is required to remove water from the oocyte as the egg swells during egg activation.

In addition, we show that the depletion of the DEG/ENaC channel, Rpk, also results in oocytes rupturing when activated. We propose that Rpk mediates optimal swelling through interactions with the cortical actin cytoskeleton, which we have previously shown to be re-organised at egg activation [23]. This hypothesis is supported by (1) coimmunoprecipitation studies in MDCK cells in which DEG/ENaC channels bind F-actin via the $\mathrm{COOH}$ terminus of $\alpha-E N a C$ and (2) mechanical pressure experiments that activate $\mathrm{DEG} / \mathrm{ENaC}$ channels resulting in the stiffening of the cortical actin in vascular endothelial cells $[47,48]$. We therefore propose that Rpk is stabilising the cortical actin to withstand the increase in volume at activation.

\section{Role of osmotic pressure and TRP channels at egg activation}

Recent work on germ-line knockout mutants in Drosophila have established the requirement of mechanosensitive Trpm channels in mediating the calcium influx at egg activation [26]. We corroborate this requirement using different mutants, RNAi and pharmacological disruption. Our data supports a model in which osmotic pressure generates tension in the plasma membrane and the cortical actin resulting in the opening of Trpm channels and subsequent calcium entry. Interestingly, the mammalian homolog TRPM3 is also activated in HEK293 cells by the application of a hypotonic solution, resulting in an intracellular calcium increase [49]. Similarly, in mammalian sensory neurones, TRPV4 and TRPV1 respond to changes in osmotic pressure [50-53]. 
311 Calcium entry mediated by TRP channels appears to be a conserved mechanism in the 312 eggs of many animals. This was first shown in Xenopus oocytes where a mechanical 313 stimulus resulted in the opening of TRPC1 [54]. More recently, mouse oocytes have been 314 shown to require TRPV3 for the calcium intracellular increase and were affected by 315 overexpression and the application of 2-APB [55]. In addition, TRPM7 was also shown to 316 be essential for the calcium influx at mouse egg activation [56]. Finally, in Caenorhabditis 317 elegans loss of the TRP3 channel resulted in a failure to show a calcium rise at egg 318 activation [57]. Taken together these examples highlight a conserved role of TRP 319 channels in mediating successful egg activation through calcium entry.

\section{The source of calcium at Drosophila egg activation}

321 Calcium waves at egg activation can be mediated by intracellular and/or external calcium 322 sources [4]. In Drosophila, Trpm regulates calcium entry across the plasma membrane 323 suggesting that the calcium source is external to the oocyte. Paradoxically, we also show 324 that external calcium is not required for a wild-type calcium wave. We argue that this data 325 is compatible and point to the perivitelline space, situated between the oocyte plasma 326 membrane and the vitelline membrane, as the calcium store. The composition of the 327 perivitelline space in the egg chamber is currently unknown. However, in the early 328 embryo, it has been shown to consist of many ions including calcium [37]. Our work 329 supports a model where the perivitelline space is pre-loaded with calcium during 330 oogenesis which enters through Trpm channels when the egg swells. This model is 331 supported by our data showing that the injection of oil (devoid of calcium) is sufficient to 332 induce a calcium rise in the oocyte.

\section{The Drosophila calcium wave is an example of a "slow" calcium wave}

334 While calcium waves can be classified by the source of ions, they can alternatively be 335 compared based on how fast they propagate [58]. In most animals, calcium waves at egg 336 activation are classified as fast, travelling at $\sim 10-30 \mu \mathrm{m} / \mathrm{sec}$ [59]. However, some wave(s) 337 propagate at $\sim 0.2-2 \mu \mathrm{m} / \mathrm{sec}$ and are classified as slow. This includes calcium influx at 338 egg activation in maize eggs which propagates at $1.13 \mu \mathrm{m} / \mathrm{sec}$ and interestingly, requires 339 mechanosensitive channels [60-62]. This is very similar to observations in Drosophila, 340 where mechanosensitive channels and the actin cytoskeleton are required for a slow 341 wave that propagates at $\sim 1.5 \mu \mathrm{m} / \mathrm{sec}$. In fact, the general mechanism for a slow calcium 342 wave [56] is strikingly similar to what we propose is occurring at Drosophila egg activation. 
343 Overall, aspects of the calcium wave, and more broadly egg activation, in Drosophila 344 appear to be conserved with a variety of other organisms. Further analysis in flies will 345 likely show even more similarities and inform our overall understanding of egg activation 346 in all species. 


\section{MATERIALS AND METHODS}

\section{Fly stocks}

349 The following fly stocks were used: UASt-myristoylated(myr)-GCaMP5); mato350 GAL4::VP16 (BL7063) and UASp-GCaMP3 [6]; tub-GAL4VP16 (Siegfried Roth); jupiter351 mCherry (Paul Conduit); me31B::GFP [63]; ripped-pocket RNAi 352 (P\{TRiP.HMS01973\}attP40, BL39053); trpm mutant (P\{EPgy2\}TrpmEY01618/CyO, 353 BL15365); trpm RNAi (BL35581 and BL44503); prip mutant (P\{SUPor-P\}PripKG08662, 354 BL14750); prip RNAi (P\{TRiP.GLC01619\}attP2, BL44464); prip RNAi 355 (P\{TRiP.HMC03097\}attP40, BL50695); deficiency (for prip) (Df(2R)BSC160/CyO, 356 BL9595). Stocks were raised on standard cornmeal-agar medium at $21^{\circ} \mathrm{C}$ or $25^{\circ} \mathrm{C}$. For 357 dissection of mature oocytes, mated females were fattened on yeast for 48 hours at $25^{\circ} \mathrm{C}$.

\section{Reagents}

359 BAPTA (Sigma-Aldrich) was used at a final concentration of $10 \mu \mathrm{M}$; BAPTA-AM + PF-127 360 (Sigma-Aldrich) was used at a final concentration of $30 \mu \mathrm{M}$; Carvacrol (Sigma-Aldrich) 361 used at $300-700 \mu \mathrm{m}$. For the above reagents, standard preparation protocols were used 362 as according to Sigma-Aldrich.

363 Activation Buffer (AB) containing $3.3 \mathrm{mM} \mathrm{NaH}_{2} \mathrm{PO}_{4}, 16.6 \mathrm{mM} \mathrm{KH}_{2} \mathrm{PO}_{4}, 10 \mathrm{mM} \mathrm{NaCl}$, $36450 \mathrm{mM} \mathrm{KCl}, 5 \%$ polyethylene glycol $8000,2 \mathrm{mM} \mathrm{CaCl}_{2}$, brought to $\mathrm{pH} 6.4$ with a 1:5 ratio 365 of $\mathrm{NaOH}: \mathrm{KOH}$ [15]; Gibco Schneider's Drosophila Medium (Thermo Fisher); Series95 366 halocarbon oil (KMZ Chemicals); EZ-Squeeze tube $125 \mu \mathrm{M}$ (Cooper Surgical). For 367 osmolarity experiments, sucrose (Sigma-Aldrich) was directly dissolved into distilled 368 water and the osmolarity was measured using an osmometer (Löser).

\section{Preparation of mature oocyte for live imaging}

370 Mature oocytes were dissected from the ovaries from fattened flies using a probe and 371 fine forceps [64]. Dissected oocytes were placed in series 95 halocarbon oil (KMZ 372 Chemicals) on $22 \times 40$ coverslips, aligned parallel to each other to maximise the 373 acquisition area for imaging, left to settle for 10 minutes, and incubated in solution ex vivo 374 [64]. 


\section{Imaging}

376 Time-series were acquired with an inverted Leica SP5, under 20x 0.7NA immersion

377 objective. The Z-stacks were acquired at $2 \mu \mathrm{m}$ steps from the first visible plane to $40 \mu \mathrm{m}$

378 deep. The Z-stacks were presented as maximum projections of the $40 \mu \mathrm{m}$ unless stated

379 otherwise.

\section{Oil injection}

381 Preparation for microinjection was carried out with a Femtotips II microinjection needle 382 (Eppendorf) and a gas pressure injection system were used to inject oil into the Stage 14 383 egg chambers [31]. Imaging was performed simultaneously with injection on a 384 DeltaVision wide-field microscope (Applied Precision) using a 20x 0.75NA numerical 385 aperture.

\section{Quantifications and analysis}

387 The calcium wave data was analysed statistically using Fisher's exact test with P-values 388 ( $P<0.05$ considered significantly different) [6]. The spindle dimensions were quantified 389 and statistically analysed using an unpaired T-test with $\mathrm{P}<0.05$ values showing significant 390 difference. The number of asterisks represents the P-value: $\left({ }^{*}\right) P \leq 0.05 ;\left({ }^{* *}\right) P \leq 0.01 ;\left({ }^{* * *}\right)$ $391 \quad P \leq 0.001$. 
393 We are grateful to Richard Parton for experimental and technical advice; Mariana 394 Wolfner, Matthias Landgraf, Howard Baylis, José Casal, Peter Lawrence for feedback, 395 discussions and advice; Richard York-Weaving for feedback on the manuscript; the 396 Zoology Imaging Facility and Matt Wayland for assistance with microscopy; Siegfried 397 Roth, Paul Conduit and Mariana Wolfner for fly stocks; and funding from the University of 398 Cambridge ISSF grant number 097814 (to TTW), Department of Zoology Balfour 399 Studentship (to AHYA), BBSRC DTP studentship (to BWW), BBSRC DTP studentship 400 (ELW), and Sir Isaac Newton Trust Research Grant (Ref 18.07ii(c)).

401 Conflicts of interest

402 The authors declare that there are no conflicts of interest. 


\section{FIGURE 1}
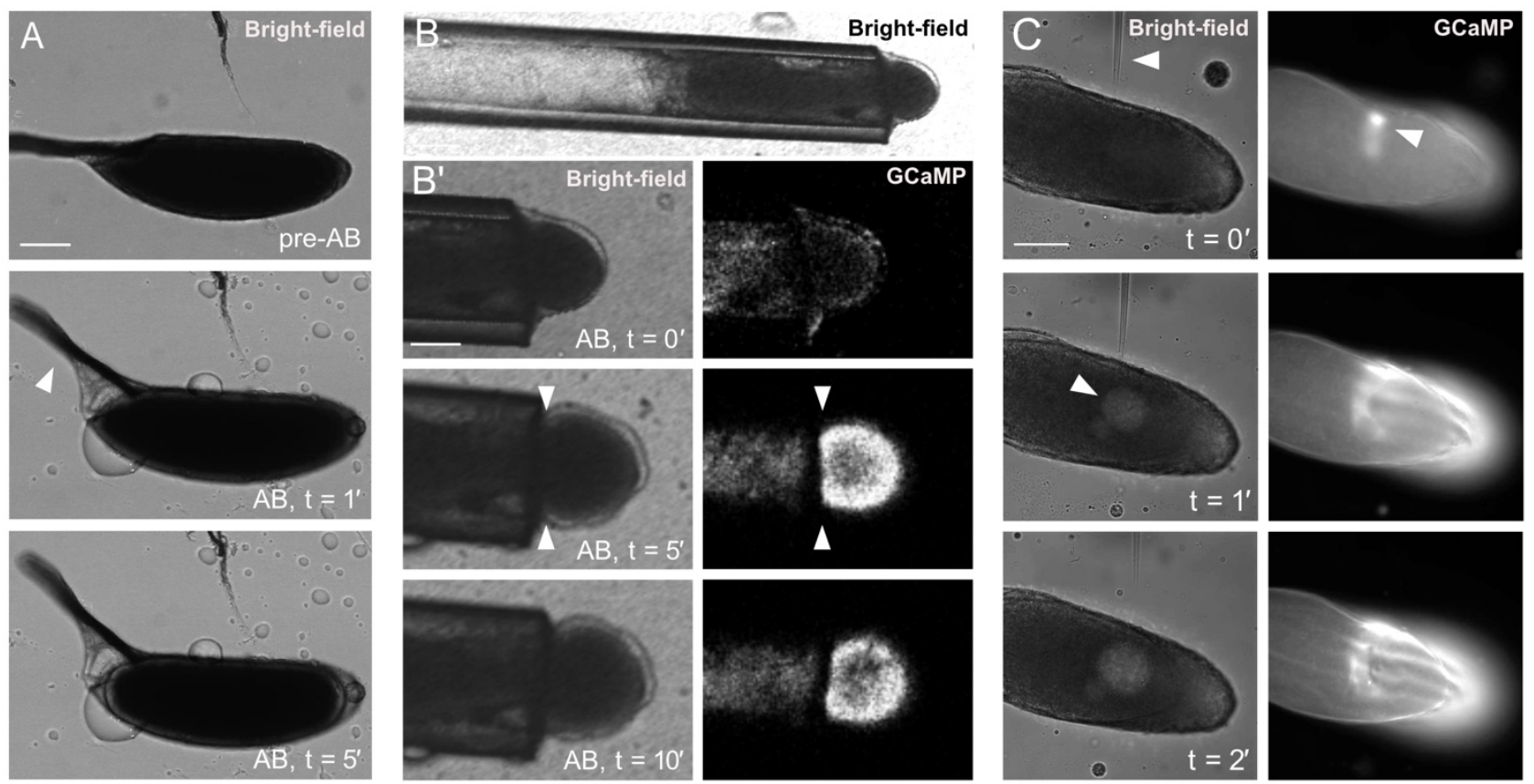

Figure 1. Egg chamber swelling is required for the initiation and propagation of the calcium wave

406 Time series showing ex-vivo mature egg chambers under bright-field $\left(A, B, B^{\prime}, C\right)$ and expressing 407 UAS-myrGCaMP5 (B,B',C). Images represent a single plane.

408 (A) Time series of a wild-type egg chamber pre- and post-addition of activation buffer (AB). Upon 409 the addition of $A B$, the egg chamber undergoes swelling, the dorsal appendages rise (white 410 arrowhead, $t=1^{\prime}$ ) and the poles become more rounded (white arrow, $t=5^{\prime}$ ). Circular droplets 411 visible on the outside of the egg are oil that was not displaced by AB. Scale bar $100 \mu \mathrm{m}$.

412 (B) Bright-field image of an egg chamber, expressing UAS-myrGCaMP5, placed in a $125 \mu \mathrm{m}$ 413 diameter tube. ( $\left.B^{\prime}\right)$ Time series of the same egg chamber in the tube, with the posterior pole 414 exposed to $A B$. The calcium wave initiates normally but does not propagate past the tube opening 415 (white arrowheads) $(n=15)$. Scale bar $60 \mu \mathrm{m}$.

416 (C) Bright-field image of a mature egg chamber, expressing UAS-myrGCaMP5, injected with 417 halocarbon oil. As the needle enters the oocyte, there is a calcium increase at the point of injection 418 (white arrowhead, $t=0^{\prime}$ ). Injected oil is seen as a circle in the cytoplasm of the egg at $t=1^{\prime}$ (white 419 arrowhead) and remains localised $\left(t=2^{\prime}\right)$. Localised swelling results in an increase in calcium, 420 which does not propagate $\left(t=2^{\prime}\right)(n=5)$. Scale bar $100 \mu \mathrm{m}$. 


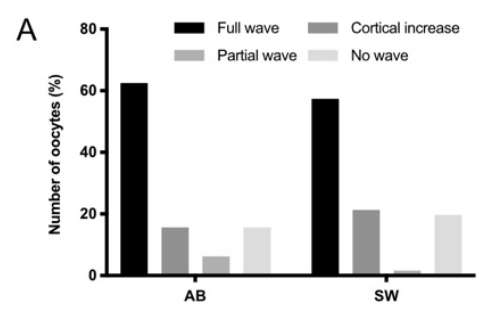

Type of solution at $260 \mathrm{mOsm}$

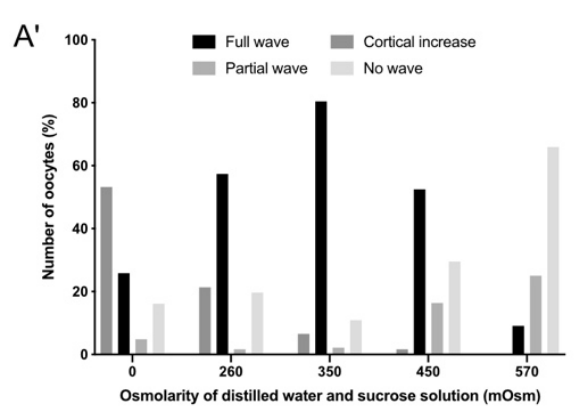

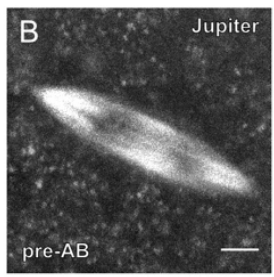
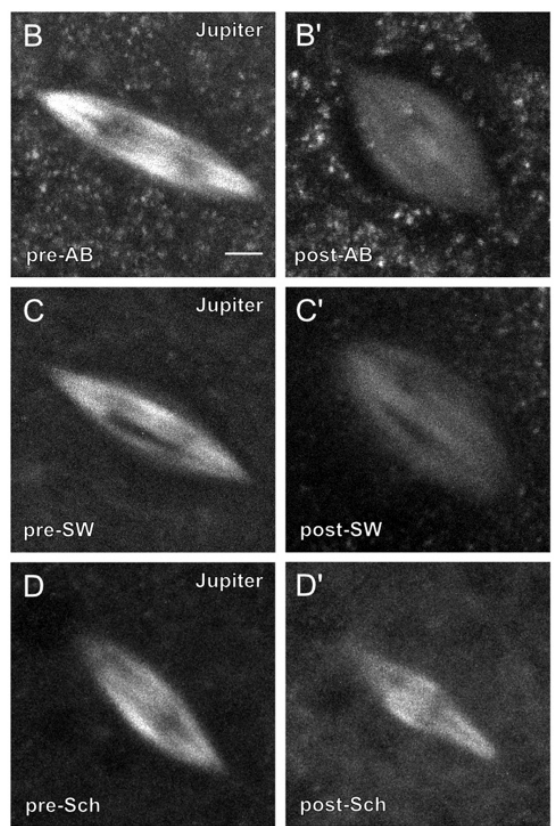
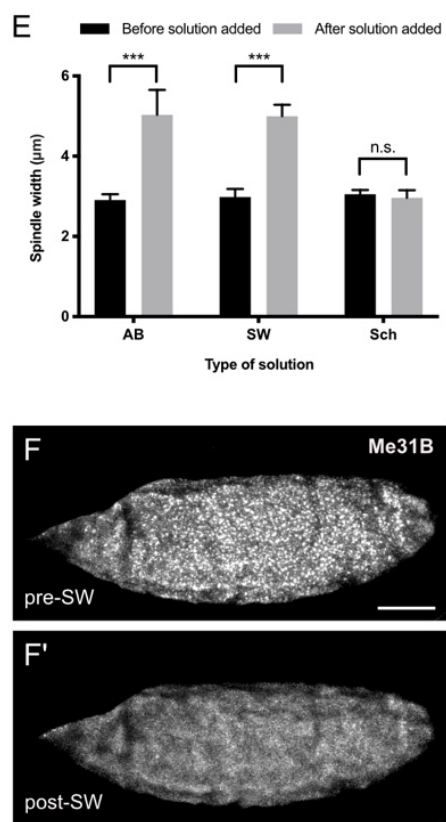

Figure 2: Osmotic pressure initiates the calcium wave and results in rearrangement of the meiotic spindle and $\mathrm{P}$ body dispersion

424 (A) Data showing activation buffer (AB) or sucrose and water (SW) of $260 \mathrm{mOsm}$ results in a 425 similar percentage of the calcium wave phenotypes when added to ex vivo egg chambers. ( $\left.A^{\prime}\right)$ 426 The data shows the number of mature oocytes activated with SW only, with a range of 427 osmolarities from $0-570$ mOsm. The number of full waves increases from $0 \mathrm{mOsm}$, peaks at 350 428 mOsm and then decreases with higher osmolarities. The proportion of egg chambers that show a cortical increase peaks at $0 \mathrm{mOsm}$ and then decreases with higher osmolarities. The proportion of partial waves increases with higher osmolarities. The proportion of no wave increases with higher osmolarities ( $n=30$ per osmolarity). This data was analysed statistically using Fisher's exact test with $\mathrm{P}<0.05$ considered significant. The proportion of full calcium waves observed at 350 mOsm is significantly higher $(P<0.05)$ than full waves at all other osmolarities shown. The proportion of cortical increases observed at 0 mOsm is significantly higher $(P<0.01)$ than cortical increases at all measured osmolarities shown. The proportion of partial waves observed at 570 mOsm is significantly higher $(P<0.01)$ than partial waves at all measured osmolarities, except 450 mOsm. The proportion of no waves observed at 570 mOsm is significantly higher $(P<0.001)$ than no waves at all other osmolarities shown.

(B-D') Mature egg chambers expressing jupiter-mCherry to visualise microtubules in the meiotic spindle. Before activation (pre) the spindle is in the shape of an ellipse, with dark regions in the middle where the DNA resides (B-D). Post-incubation images were taken 10 minutes after the addition of the solution. The spindle shows an increase in width following the addition of $A B$ and SW (260 mOsm) (B-C'), however, the width does not change upon the addition of Schneider's 
445 (E) Graph showing a change in spindle width upon addition of $A B$, SW and Sch. The data was 446 analysed statistically using an unpaired T-test with $\mathrm{P}<0.05$ considered significant. The spindle 447 shows a significant increase in width by $2.1 \mu \mathrm{m}(\mathrm{a} 1.7 \mathrm{x}$ increase $)(\mathrm{P}<0.001)$ upon the addition of 448 AB or SW (260 mOsm). There is no significant change in width upon the addition of Sch $(n=15$ 449 per solution).

450 (F-F') Time-series of ex vivo egg chamber expressing me31B::GFP following the addition of SW 451 (260 mOsm). P bodies appear as granular puncta pre-SW and disperse following the addition of $452 \mathrm{SW}$, consistent with the addition of $A B(n=15)$. Post-incubation images were taken at 10 minutes 453 after incubation of solution. Scale bar $60 \mu \mathrm{m}$. Maximum projection $40 \mu \mathrm{m}$. 
A
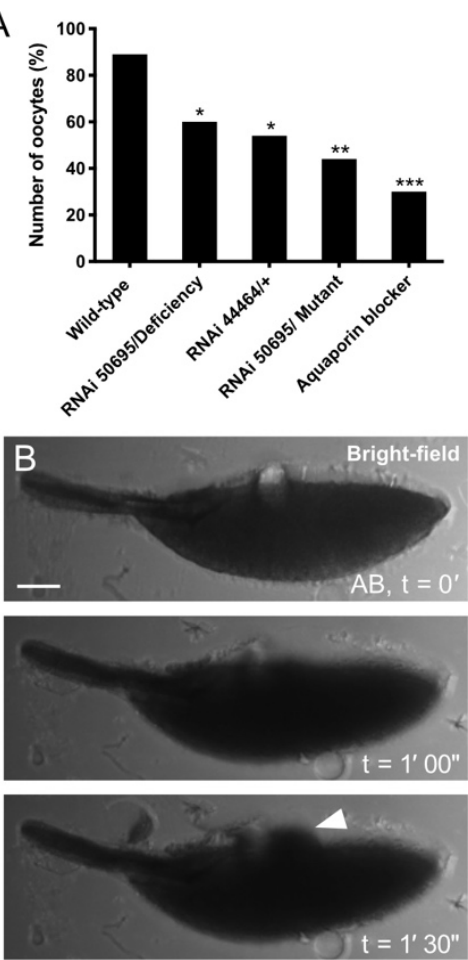
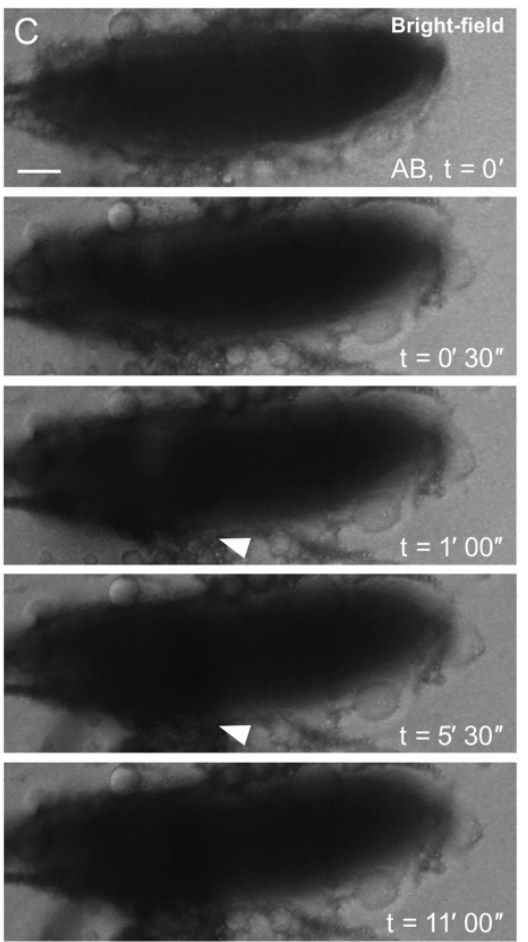
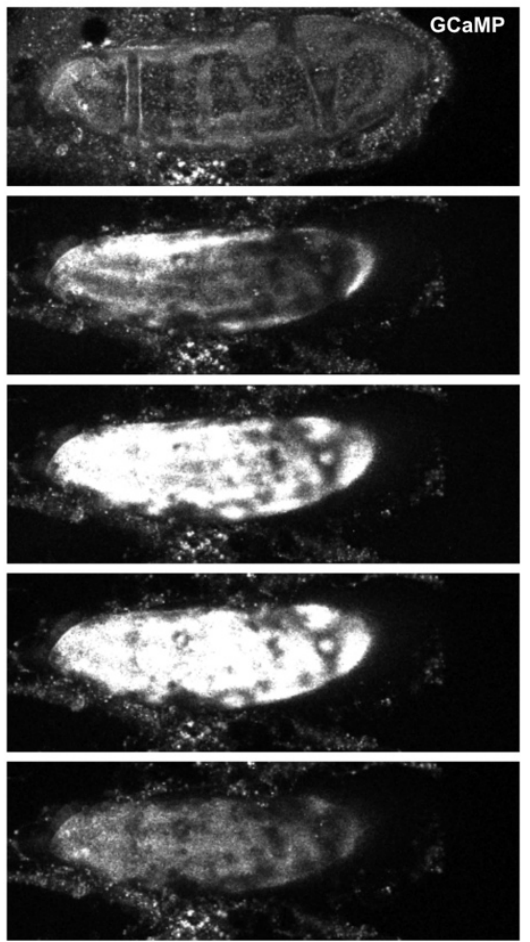

Figure 3. Water homeostasis is required for egg activation

456 (A) The data shows the presence of the calcium wave in Aquaporin depleted backgrounds. 457 Aquaporin depletion was achieved through knockdown using BL50695 (germline) and BL44464 458 (germline and somatic) RNAi, deficiency (Df(2R)BSC160/Cyo), prip mutant (y1; P\{SUPor459 P\}PripKG08662) and the broad Aquaporin channel antagonist copper sulfate. Upon the addition 460 of $A B$, the number of oocytes with the calcium wave significantly decreased to $50 \%$ in the germline 461 knockdown over the deficiency $(n=25, P<0.05)$ or mutant $(n=18, P<0.001)$. A similar significant 462 decrease was also observed with only one copy knock-down of both somatic and germline Prip $463(B L 44464)(n=13, p<0.05)$. Addition of copper sulfate results in a significant decrease of waves 464 to approximately $30 \%(n=44, P<0.001)$.

465 (B) Bright-field time series of mature egg chamber in an Aquaporin depleted background (RNAi $46650695 /$ deficiency). Upon addition of $A B, 50 \%$ of the oocytes burst compared to $3 \%$ in the wild467 type, with the cytoplasm leaking within 1 minute and 30 seconds (white arrowhead) $(n=120)$. 468 Scale bar $60 \mu \mathrm{m}$. Maximum projection $40 \mu \mathrm{m}$.

469 (C) Time-series of ex vivo mature egg chamber expressing UAS-myrGCaMP5 and two copies of 470 ripped-pocket $\mathrm{RNAi}$ following the addition of $\mathrm{AB}$. The cortical increase appears within 30 seconds 471 of the addition of $A B$, which is followed by the oocyte burst and cytoplasm leaking out in $74 \%$ of 472 egg chambers (white arrowhead). The dark spots represent excess tissue and oil droplets. Scale 473 bar $60 \mu \mathrm{m}$. Maximum projection $40 \mu \mathrm{m}$. 

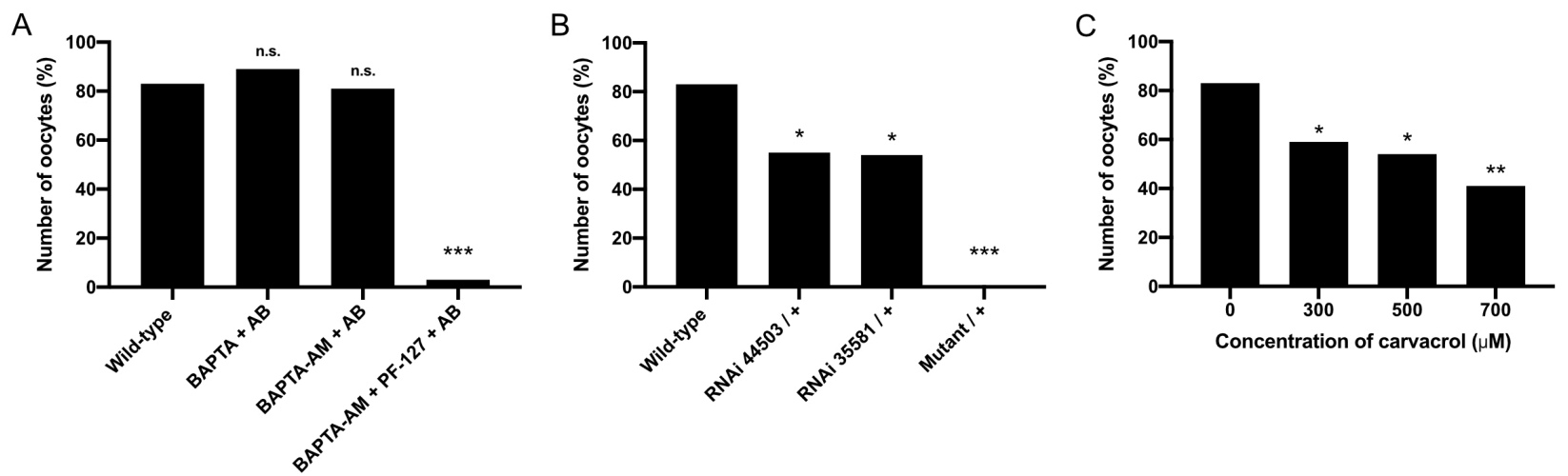

\section{Figure 4. Internal calcium and Trpm channel are required for the calcium wave}

(A) The graph shows the presence of the calcium wave in external or internal calcium-depleted backgrounds. Depletion of external calcium was achieved through the addition of calcium chelator BAPTA or BAPTA-AM in AB. Depletion of internal calcium was achieved through the addition of BAPTA-AM and PF-127 in AB. Upon the addition of BAPTA or BAPTA-AM in AB, there was no significant difference in the number of oocytes with the calcium wave ( $n=19$ and $n=57$ ). The addition of BAPTA-AM and PF-127 in AB resulted in a significant decrease in the number of calcium waves $(n=34, P<0.001)$. Data was statistically analysed using Fisher's exact test.

(B-C) The graphs show the presence of the calcium waves in Trpm depleted backgrounds. Trpm depletion was achieved through knockdown using BL44503 (somatic and germline) and BL35581 (germline) RNAi, trpm mutant (y1 w67c23; P\{EPgy2\}TrpmEY01618/CyO) (B) and (C) the broad Trpm blocker carvacrol. Upon the addition of $A B(B)$, the number of the oocytes with the calcium wave significantly decreased with only one copy knockdown of both somatic and germline Trpm (BL44503) $(n=96, P<0.01)$ and germline only $(B L 35581)(n=35, P<0.05)$. A significant decrease in the number of the calcium waves was also observed in Trpm mutant background $(n=14$, $P<0.001)$. (C) A significant decrease in the number of the calcium waves was also observed with the addition of $A B$ with the broad Trpm blocker carvacrol in a concentration-dependent manner of $300 \mu \mathrm{M}(\mathrm{n}=27, \mathrm{P}<0.05), 500 \mu \mathrm{M}(\mathrm{n}=24, \mathrm{P}<0.05)$ and $700 \mu \mathrm{M}(\mathrm{n}=24, \mathrm{P}<0.01)$. Data was statistically analysed using Fisher's exact test. 


\section{FIGURE 5}

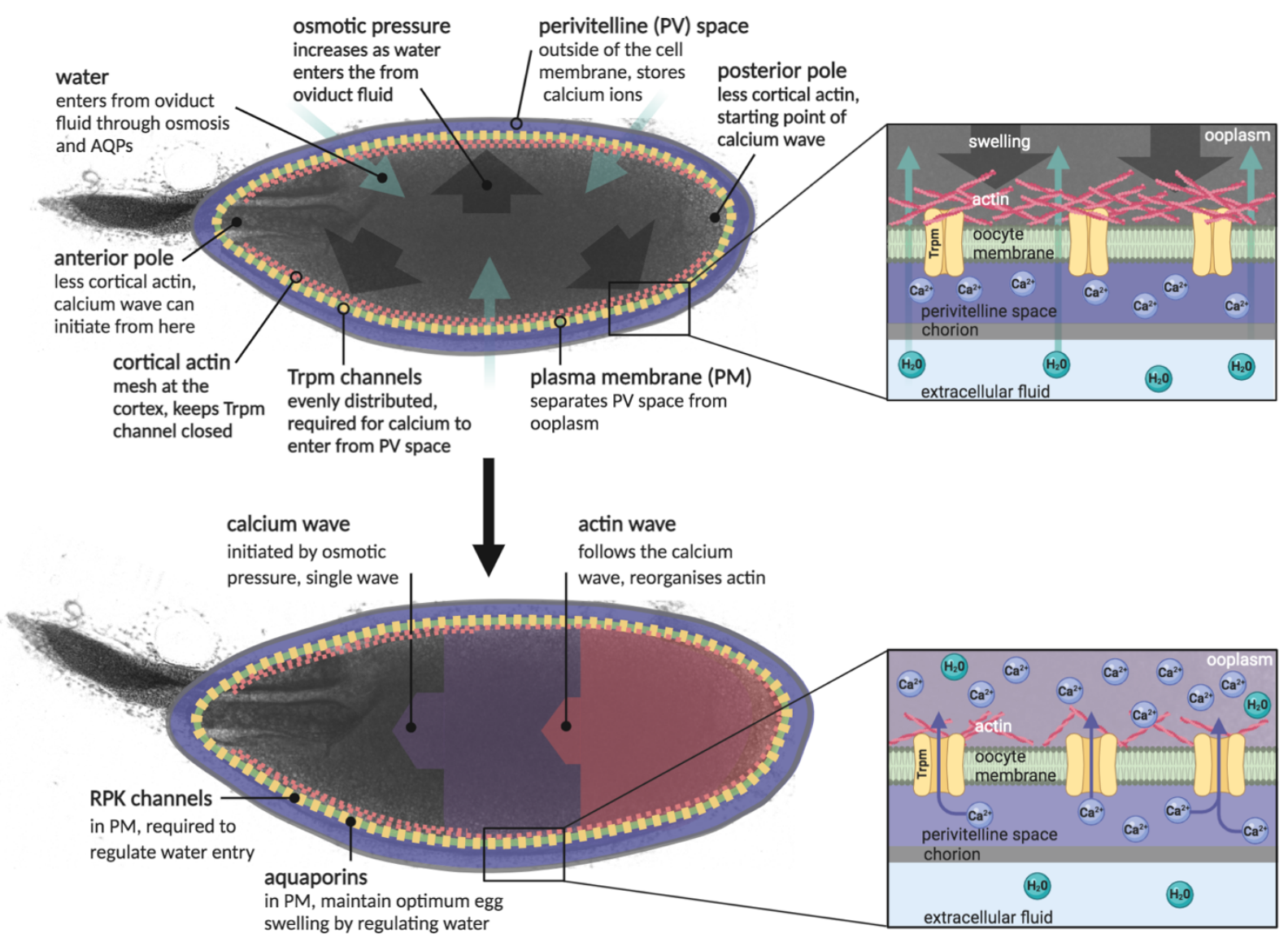

495 Figure 5. Model of Drosophila egg activation

496 Essential components and processes of Drosophila egg activation are outlined in the panels. A comprehensive description of the model is included in the discussion. Created with BioRender.com. 


\section{REFERENCES}

500 1. Whitaker M. 2006 Calcium at Fertilization and in Early Development. Physiol Rev 86, $501 \quad$ 25--88. (doi:10.1152/physrev.00023.2005)

502 2. Horner VL, Wolfner MF. 2008 Transitioning from egg to embryo: Triggers and mechanisms of egg activation. Dev Dynam 237, 527--544. (doi:10.1002/dvdy.21454)

3. Swann K, Lai FA. 2016 Egg Activation at Fertilization by a Soluble Sperm Protein. Physiol Rev 96, 127--149. (doi:10.1152/physrev.00012.2015)

4. Stricker SA. 1999 Comparative Biology of Calcium Signaling during Fertilization and Egg Activation in Animals. Dev Biol 211, 157--176. (doi:10.1006/dbio.1999.9340)

5. York-Andersen AH, Parton RM, Bi CJ, Bromley CL, Davis I, Weil TT. 2015 A single and rapid calcium wave at egg activation in Drosophila. Biol Open 4, 553--560. (doi:10.1242/bio.201411296)

6. Kaneuchi T, Sartain CV, Takeo S, Horner VL, Buehner NA, Aigaki T, Wolfner MF. 2015 Calcium waves occur as Drosophila oocytes activate. Proc National Acad Sci 112, 791--796. (doi:10.1073/pnas.1420589112)

7. Parrington J, Davis LC, Galione A, Wessel G. 2007 Flipping the switch: How a sperm activates the egg at fertilization. Dev Dynam 236, 2027--2038.

516 (doi:10.1002/dvdy.21255)

517 8. Horner VL, Wolfner MF. 2008 Mechanical stimulation by osmotic and hydrostatic pressure activates Drosophila oocytes in vitro in a calcium-dependent manner. Dev Biol 316, 100--109. (doi:10.1016/j.ydbio.2008.01.014)

520 9. Kishimoto T. 1998 Cell cycle arrest and release in starfish oocytes and eggs. Semin 521 Cell Dev Biol 9, 549--557. (doi:10.1006/scdb.1998.0249)

10. Harada K, Oita E, Chiba K. 2003 Metaphase I arrest of starfish oocytes induced via the MAP kinase pathway is released by an increase of intracellular $\mathrm{pH}$. Development 130, 4581--4586. (doi:10.1242/dev.00649)

11. Lindsay LL, Hertzler PL, Clark WH. 1992 Extracellular Mg2+ induces an intracellular Ca2+ wave during oocyte activation in the marine shrimp Sicyonia ingentis. Dev Biol 152, 94--102. (doi:10.1016/0012-1606(92)90159-e)

12. Went DF. 1982 Egg Activation and Parthenogenetic Reproduction in Insects. Biol Rev 57, 319--344. (doi:10.1111/j.1469-185x.1982.tb00371.x) 
14. Went DF, Krause G. 1974 Alteration of egg architecture and egg activation in an endoparasitic Hymenopteran as a result of natural or imitated oviposition. Wilhelm Roux' Archiv Für Entwicklungsmechanik Der Org 175, 173--184. (doi:10.1007/bf00582090)

15. Mahowald AP, Goralski TJ, Caulton JH. 1983 In vitro activation of Drosophila eggs. Dev Biol 98, 437--445. (doi:10.1016/0012-1606(83)90373-1)

16. Yamamoto DS, Hatakeyama M, Matsuoka H. 2013 Artificial activation of mature unfertilized eggs in the malaria vector mosquito, Anopheles stephensi (Diptera, Culicidae). J Exp Biol 216, 2960--2966. (doi:10.1242/jeb.084293)

17. Oishi K, Sawa M, Hatakeyama M, Kageyama Y. 1993 Genetics and biology of the sawfly,Athalia rosae (Hymenoptera). Genetica 88, 119--127. (doi:10.1007/bf02424468)

18. Tojo K, Machida R. 1998 Early embryonic development of the mayfly Ephemera japonica McLachlan (Insecta: Ephemeroptera, Ephemeridae). J Morphol 238, 327--335. (doi:10.1002/(sici)1097-4687(199812)238:3<327::aid-jmor4>3.0.co;2-j)

19. Li J. 1994 Egg chorion tanning in Aedes aegypti mosquito. Comp Biochem Physiology Part Physiology 109, 835--843. (doi:10.1016/0300-9629(94)90231-3)

20. Li JS, Li J. 2006 Major chorion proteins and their crosslinking during chorion hardening in Aedes aegypti mosquitoes. Insect Biochem Molec 36, 954--964. (doi:10.1016/j.ibmb.2006.09.006)

21. Doane WW. 1960 Completion of Meiosis in Uninseminated Eggs of Drosophila melanogaster. Science 132, 677--678. (doi:10.1126/science.132.3428.677)

22. Sartain CV, Wolfner MF. 2013 Calcium and egg activation in Drosophila. Cell Calcium 53, 10--15. (doi:10.1016/j.ceca.2012.11.008)

23. York-Andersen AH, Hu Q, Wood BW, Wolfner MF, Weil TT. 2020 A calciummediated actin redistribution at egg activation in Drosophila. Mol Reprod Dev 87, 293-304. (doi:10.1002/mrd.23311)

24. Lin H, Spradling AC. 1993 Germline Stem Cell Division and Egg Chamber Development in Transplanted Drosophila Germaria. Dev Biol 159, 140--152. (doi:10.1006/dbio.1993.1228)

25. Hu Q, Vélez-Avilés AN, Wolfner MF. 2020 Drosophila Plc21C is involved in calcium wave propagation during egg activation. Micropublication Biology 2020, 10.17912/micropub.biology.000235. (doi:10.17912/micropub.biology.000235)

26. Hu Q, Wolfner MF. 2019 The Drosophila Trpm channel mediates calcium influx during egg activation. Proc National Acad Sci 116, 18994--19000.

(doi:10.1073/pnas.1906967116) 
27. Hu Q, Wolfner MF. 2020 Regulation of Trpm activation and calcium wave initiation during Drosophila egg activation. Mol Reprod Dev 87, 880--886.

(doi:10.1002/mrd.23403)

28. Endow SA, Komma DJ. 1997 Spindle Dynamics during Meiosis in Drosophila Oocytes. J Cell Biology 137, 1321--1336. (doi:10.1083/jcb.137.6.1321)

29. Page AW, Orr-Weaver TL. 1997 Activation of the Meiotic Divisions in Drosophila Oocytes. Dev Biol 183, 195--207. (doi:10.1006/dbio.1997.8506)

30. Heifetz Y, Yu J, Wolfner MF. 2001 Ovulation Triggers Activation of Drosophila Oocytes. Dev Biol 234, 416--424. (doi:10.1006/dbio.2001.0246)

31. Weil TT et al. 2012 Drosophila patterning is established by differential association of mRNAs with P bodies. Nat Cell Biol 14, 1305--1313. (doi:10.1038/ncb2627)

32. Verkman AS. 2011 Aquaporins at a glance. J Cell Sci 124, 2107--2112. (doi:10.1242/jcs.079467)

33. Verkman AS, Anderson MO, Papadopoulos MC. 2014 Aquaporins: important but elusive drug targets. Nat Rev Drug Discov 13, 259--277. (doi:10.1038/nrd4226)

34. Chalfie M, Wolinsky E. 1990 The identification and suppression of inherited neurodegeneration in Caenorhabditis elegans. Nature 345, 410--416.

(doi:10.1038/345410a0)

35. Driscoll M, Chalfie M. 1991 The mec-4 gene is a member of a family of Caenorhabditis elegans genes that can mutate to induce neuronal degeneration. Nature 349, 588--593. (doi:10.1038/349588a0)

36. García-Añoveros J, Ma C, Chalfie M. 1995 Regulation of Caenorhabditis elegans degenerin proteins by a putative extracellular domain. Curr Biol 5, 441--448. (doi:10.1016/s0960-9822(95)00085-6)

37. Meer JM van der, Jaffe LF. 1983 Elemental composition of the perivitelline fluid in early Drosophila embryos. Dev Biol 95, 249--252. (doi:10.1016/0012-1606(83)90025-8)

38. Bellen HJ et al. 2004 The BDGP Gene Disruption Project Single Transposon Insertions Associated With 40 of Drosophila Genes. Genetics 167, 761--781. (doi:10.1534/genetics.104.026427)

39. Hofmann T, Chubanov V, Chen X, Dietz AS, Gudermann T, Montell C. 2010 Drosophila TRPM Channel Is Essential for the Control of Extracellular Magnesium Levels. Plos One 5, e10519. (doi:10.1371/journal.pone.0010519) Hofmann T, Chubanov V, Chen X, Dietz AS, Gudermann T, Montell C. 2010 Drosophila TRPM Channel Is Essential for the Control of Extracellular Magnesium Levels. Plos One 5, e10519. (doi:10.1371/journal.pone.0010519) 
40. Chubanov V, Schäfer S, Ferioli S, Gudermann T. 2014 Natural and Synthetic Modulators of the TRPM7 Channel. Cells 3, 1089--1101. (doi:10.3390/cells3041089)

41. O'Connor E, Kimelberg H. 1993 Role of calcium in astrocyte volume regulation and in the release of ions and amino acids. J Neurosci 13, 2638--2650.

(doi:10.1523/jneurosci.13-06-02638.1993)

42. MacLeod RJ, Hamilton JR. 1999 Increases in Intracellular pH and Ca2+ are Essential for K+ Channel Activation After Modest 'Physiological' Swelling in Villus Epithelial Cells. J Membr Biology 172, 47--58. (doi:10.1007/s002329900582)

43. Weskamp M, Seidl W, Grissmer S. 2000 Characterization of the Increase in [Ca2+]i During Hypotonic Shock and the Involvement of $\mathrm{Ca2+-activated} \mathrm{K}+$ Channels in the Regulatory Volume Decrease in Human Osteoblast-like Cells. J Membr Biology 178, 11 -20. (doi:10.1007/s002320010010)

44. Shen M, Chou C, Browning JA, Wilkins RJ, Ellory JC. 2001 Human cervical cancer cells use Ca2+ signalling, protein tyrosine phosphorylation and MAP kinase in regulatory volume decrease. J Physiology 537, 347--362. (doi:10.1111/j.14697793.2001.00347.x)

45. Kültz D, Burg MB. 1998 Intracellular Signaling in Response to Osmotic Stress. Contrib Nephrol 123, 94--109. (doi:10.1159/000059923)

46. Kliewer JW. 1961 Weight and Hatchability of Aedes aegypti Eggs (Diptera: Culicidae)1. Ann Entomol Soc Am 54, 912--917. (doi:10.1093/aesa/54.6.912)

47. Mazzochi C, Bubien JK, Smith PR, Benos DJ. 2006 The Carboxyl Terminus of the a-Subunit of the Amiloride-sensitive Epithelial Sodium Channel Binds to F-actin. J Biol Chem 281, 6528--6538. (doi:10.1074/jbc.m509386200)

48. Kusche-Vihrog K, Urbanova K, Blanqué A, Wilhelmi M, Schillers H, Kliche K, Pavenstädt H, Brand E, Oberleithner H. 2011 C-Reactive Protein Makes Human Endothelium Stiff and Tight. Hypertension 57, 231--237. (doi:10.1161/hypertensionaha.110.163444)

49. Grimm C, Kraft R, Sauerbruch S, Schultz G, Harteneck C. 2003 Molecular and Functional Characterization of the Melastatin-related Cation Channel TRPM3. J Biol Chem 278, 21493--21501. (doi:10.1074/jbc.m300945200)

50. Liedtke W, Choe Y, Martí-Renom MA, Bell AM, Denis CS, AndrejŠali, Hudspeth AJ, Friedman JM, Heller S. 2000 Vanilloid Receptor-Related Osmotically Activated Channel (VR-OAC), a Candidate Vertebrate Osmoreceptor. Cell 103, 525--535. (doi:10.1016/s0092-8674(00)00143-4)

51. Strotmann R, Harteneck C, Nunnenmacher K, Schultz G, Plant TD. 2000 OTRPC4, a nonselective cation channel that confers sensitivity to extracellular osmolarity. Nat Cell Biol 2, 695--702. (doi:10.1038/35036318) 
52. Naeini RS, Witty M-F, Séguéla P, Bourque CW. 2006 An N-terminal variant of Trpv1 channel is required for osmosensory transduction. Nat Neurosci 9, 93--98. (doi:10.1038/nn1614)

53. Ciura S, Liedtke W, Bourque CW. 2011 Hypertonicity Sensing in Organum Vasculosum Lamina Terminalis Neurons: A Mechanical Process Involving TRPV1 But Not TRPV4. J Neurosci 31, 14669--14676. (doi:10.1523/jneurosci.1420-11.2011)

54. Methfessel C, Witzemann V, Takahashi T, Mishina M, Numa S, Sakmann B. 1986 Patch clamp measurements onnXenopus laevis oocytes: currents through endogenous channels and implanted acetylcholine receptor and sodium channels. Pflügers Archiv 407, 577--588. (doi:10.1007/bf00582635)

55. Lee HC, Yoon S-Y, Lykke-Hartmann K, Fissore RA, Carvacho I. 2016 TRPV3 channels mediate Ca2+ influx induced by 2-APB in mouse eggs. Cell Calcium 59, 21-31. (doi:10.1016/j.ceca.2015.12.001)

56. Carvacho I, Ardestani G, Lee HC, McGarvey K, Fissore RA, Lykke-Hartmann K. 2016 TRPM7-like channels are functionally expressed in oocytes and modulate postfertilization embryo development in mouse. Sci Rep-uk 6, 34236. (doi:10.1038/srep34236)

57. Takayama J, Onami S. 2016 The Sperm TRP-3 Channel Mediates the Onset of a Ca2+ Wave in the Fertilized C. elegans Oocyte. Cell Reports 15, 625--637. (doi:10.1016/j.celrep.2016.03.040)

58. Jaffe LF. 2008 Calcium waves. Philosophical Transactions Royal Soc B Biological Sci 363, 1311--1317. (doi:10.1098/rstb.2007.2249)

59. Jaffe LF. 2002 On the conservation of fast calcium wave speeds. Cell Calcium 32 , 217--229. (doi:10.1016/s0143416002001574)

60. Digonnet C, Aldon D, Leduc N, Dumas C, Rougier M. 1997 First evidence of a calcium transient in flowering plants at fertilization. Dev Camb Engl 124, 2867-74.

61. Antoine AF, Faure J-E, Cordeiro S, Dumas C, Rougier M, Feijó JA. 2000 A calcium influx is triggered and propagates in the zygote as a wavefront during in vitro fertilization of flowering plants. Proc National Acad Sci 97, 10643--10648. (doi:10.1073/pnas.180243697)

62. Antoine AF, Dumas C, Faure J-E, Feijó JA, Rougier M. 2001 Egg activation in flowering plants. Sex Plant Reprod 14, 21--26. (doi:10.1007/s004970100088)

63. Nakamura A, Amikura R, Hanyu K, Kobayashi S. 2001 Me31B silences translation of oocyte-localizing RNAs through the formation of cytoplasmic RNP complex during Drosophila oogenesis. Dev Camb Engl 128, 3233-42. 
bioRxiv preprint doi: https://doi.org/10.1101/2021.02.16.431163; this version posted February 17, 2021. The copyright holder for this preprint (which was not certified by peer review) is the author/funder. All rights reserved. No reuse allowed without permission.

675 64. Derrick CJ, York-Andersen AH, Weil TT. 2016 Imaging Calcium in Drosophila at 676 Egg Activation. J Vis Exp (doi:10.3791/54311) 